\title{
Least-squares color Halftoning Algorithm Based on Clustering Analysis
}

\author{
Zifen $\mathrm{He}^{1, \mathrm{a}}$, Yinhui Zhang ${ }^{1, \mathrm{~b}^{*}}$ \\ ${ }^{1}$ Kunming University of Science and Technology,Faculty of mechanical and electrical engineering, \\ Kunming, 650500, China
}

azyhhzf1998@163.com, byinhui_z@163.com

\begin{abstract}
Keywords:Color halftoning;Human vision system(HVS);Printer model;Clustering Analysis;Least squares
\end{abstract}

\begin{abstract}
We presents a new method for color printers. Our algorithm applies to both a color printer model and a model for the human visual system (HVS). The method strives to minimize the perceived error between the continuous original image and the halftone image. The color printer models can account for a variety of printer characteristics. We propose a specific printer model that accounts for overlap between neighboring dots of ink and spectral absorption properties of the inks. The color image is partitioned into several regions using clustering segmentation method, whose performance depends on the selection of distance metrics. Each clustering uses the least-squares model-based(Lsmb) algorithm to obtain halftone image. A performance measure for halftone images is used to evaluate our algorithm. Analysis and simulation results show that the proposed algorithm produces better color halftone image quality when we increase the number of clustering with a certain range.
\end{abstract}

\section{Introduction}

Digital color halftoning transforms a continuous-tone color image into an image with a limited number of colors for printing. The importance of this process arises from the fact that many color imaging systems use output devices such as color printers and low-bit depth displays. Examples include converting a 24-bit color image to a 3-bit color image.

Color Halftoning is a method for creating the illusion of continuous tone output with a low-bit device. Effective digital color halftoning can substantially improve the quality of rendered images at minimal cost.[1] The halftoning algorithms can be broadly classified into three categories[2]. These include ordered dithering, error diffusion and optimization-based techniques. In screening, approaches used to prevent these artifacts include minimizing color or brightness fluctuation [3]or to create halftones with a desired frequency content such as blue noise [4] and green noise [5]. Flohr et al. used the total squared error in a luminance/chrominance-based space as the metric for their model-based color halftoning algorithm[6] . The error criterion of this approach provides a measure of halftone image quality that takes into account the characteristics of both the display device (printer) and the human visual system and can be used to evaluate the performance of any halftoning technique[7].

\section{Color Print Model}

The role of a color printer model is to predict gray levels produced by printer and easy to incorporate in halftoning algorithm. A circular dot-overlap model were developed for B\&W printers that accounts for the dot-overlap characteristic like that illustrated in Fig. 1. In the B\&W case, all the dots have the same color[8]. In the color case, the overlapping segments can take different colors depending on the color of the neighboring dots. Fig. 2 shows the segments of different colors that we can get within a pixel. 


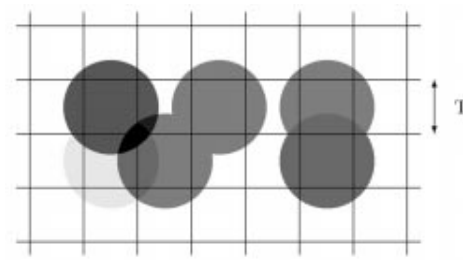

Fig.1 Color-dot Overlap

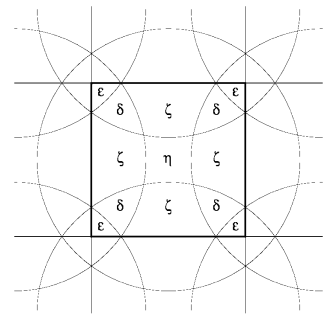

Fig.2 Overlapping Segments of Color

The color of each segment can be determined independently for each of the color components. This is because cyan ink affects only the red component, magenta affects only the green, and yellow affects only the blue. The black is not necessary in this case, since a combination of the other three inks produces a perfect black. Thus, the average color for the site can be determined independently for each color component, and the color printer model takes the

$$
p_{i, j}=\left(P_{i, j}^{R}, P_{i, j}^{G}, P_{i, j}^{B}\right)=\left(P^{R}\left(W_{i, j}^{C}\right), P^{G}\left(W_{i, j}^{M}\right), P^{B}\left(W_{i, j}^{Y}\right)\right)
$$

\section{Color Human Visual System Model}

The color/tone discrimination and appearance closely depend on spatial pattern in addition to the global differences in perceptual attributes like hue, saturation, and brightness. This finding has motivated the use of color human visual system (HVS) models for design and evaluation of the performance of digital halftoning algorithms, which aim to minimize the visual differences between continuous-tone images and their halftones. Zhang and Wandell used an opponent channel representation based HVS model called S-CIELAB to predict the perceived quality of color halftone images [9].

$$
Y_{y}=116 \frac{Y}{Y_{n}}-16 \quad C_{x}=500\left[\frac{X}{X_{n}}-\frac{Y}{Y_{n}}\right] \quad C_{z}=200\left[\frac{Y}{Y_{n}}-\frac{Z}{Z_{n}}\right]
$$

\section{Least-squares Model Halftoning}

The color Lsmb algorithm attempts to produce an optimal halftoned reproduction, by minimizing the squared error between the response of the cascade of the printer and visual models to the binary image and the response of the visual model to the original color image.

In this article, we will use the following notation. Suppose we are given a color image $\left[x_{i, j}\right]$, where $x_{i, j}$ denotes the pixel located at the $i$-th column and the $j$-th row of a grid. The color level of each pixel varies from 0 equal to white to 1 equal to black. Assuming that the image has been sampled so there is one pixel per dot to be generated. Thus the color image array $\left[x_{i, j}\right]$ and the binary image array $\left[b_{i, j}\right]$ have the same dimensions. We are also given a printer model with the sliding-window form and an eye model of the form with a memoryless nonlinearity $n($.$) followed by an FIR filter with impulse$ response $\left[h_{i, j}\right]$. In the color Lsmb approach we seek the halftone image that minimizes the squared error.

$$
\begin{aligned}
& E=\sum_{i, j}\left\|z_{i, j}-w_{i, j}\right\|^{2} \\
& z_{i, j}=x_{i, j} * g_{i, j}^{\prime} \\
& w_{i, j}=P\left(W_{i, j}\right) * g_{i, j}
\end{aligned}
$$

Note that we have allowed different impulse responses $g_{i, j}, g_{i, j}$ for the eye filters corresponding to the halftone and continuous-tone images. The boundary conditions assume that no ink is placed outside the image borders. The least-squares solution can be obtained by iterative techniques. In this 
paper, we will use the iterative scheme that was used an eye filter designed for the viewing distances of 24 inches and a printer resolution of 300dpi, the starting point obtained is a random start.

\section{Image Segmentation using Clustering}

The simplest form of clustering is partition clustering which aims at partitioning a given data set into disjoint subsets(clusters) so that specific clustering criteria are optimized. The most widely used criterion is the clustering error criterion which for each point computes its squared distance from the corresponding cluster center and then takes the sum of these distances for all points in the data set. A popular clustering method that minimizes the clustering error is the K-means algorithm.

An algorithm for partitioning (or clustering) $N$ data points into $K$ disjoint subsets $S_{j}$ containing $N_{j}$ data points so as to minimize the sum-of-squares criterion.

$$
J=\sum_{j=1}^{K} \sum_{n \in S_{j}}\left|x_{n}-\mu_{j}\right|^{2}
$$

where $x_{n}$ is a vector representing the $n$th data point and $\mu_{j}$ is the geometric centroid of the data points in $S_{j}$. In general, the algorithm does not achieve a global minimum of $J$ over the assignments. In fact, since the algorithm uses discrete assignment rather than a set of continuous parameters, the "minimum" it reaches cannot even be properly called a local minimum. Despite these limitations, the algorithm is used fairly frequently as a result of its ease of implementation.

\section{Experimental Results and Discussion}

In this paper, we use the Lena color image of sizes $256 \times 256$ to demonstrate the experimental results. All halftones are generated and printed at a resolution of 300dpi and 24inches viewing distance. In the experiment, we compare two algorithms: color Lsmb and the proposed algorithms.

Firstly, we use K-means clustering that needs to determine the number of cluster classes and distance measurements which to determine the degree of similarity between pixels and classes. The gray image is partitioned into two, three and four regions using K-means image segmentation method. Each label shows a different class. The results are shown in Fig. 3.

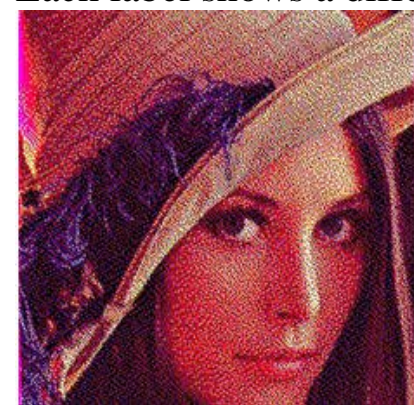

(a)

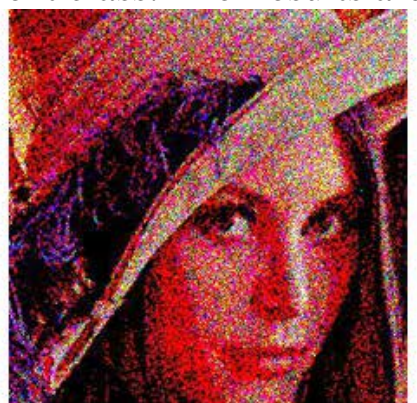

(b)

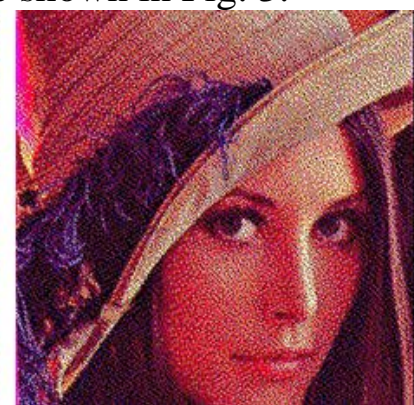

(c)

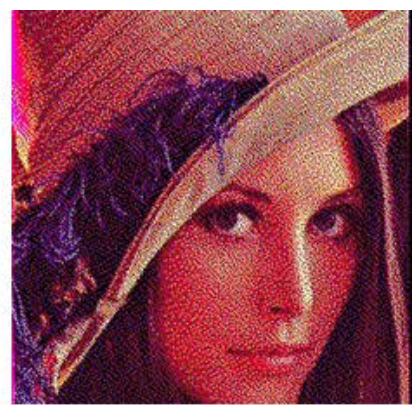

(d)

Fig. 3 Color Lsmb Halftoning and Proposed Algorithm in k (a)color Lsmb (2)k=2 (c)k=3 (d)k=4

Fig. 3 show the halftone of Lena generated using the least-squares color model-based halftoning with an eye filter designed for the viewing distances of 24 inches and a printer resolution of 300dpi, the starting point obtained is a random start. On testing with the image, we founded that when comparing the quality measurements of halftone images with MSEv, MSNR and PSNR. The test results are shown in Table 1. 
Table 1. Quality measurement of halftones with different algorithms in terms of MSEv, WSNR and PSNR when $\mathrm{k}=2,3$ and 4

\begin{tabular}{|c|c|c|c|c|c|}
\hline Image & Algorithm & Cluster & MSEv & WSNR(dB) & PSNR(dB) \\
\hline Lena & Color Lsmb & & 0.2391 & 23.7185 & 26.79 \\
\hline & Proposed & 2 & 0.2125 & 24.6992 & 26.95 \\
\hline & & 3 & 0.1494 & 24.9698 & 27.76 \\
\hline
\end{tabular}

Table1 lists MSEv, WSNR and PSNR figures for the test images with color Lsmb and proposed algorithm. We plot the MSEv, WSNR and PSNR for the test images in Fig. 4.
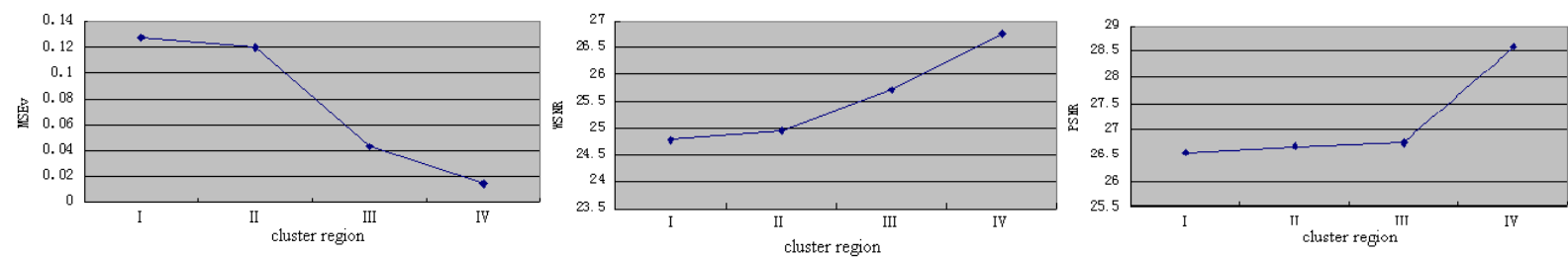

Fig. 4 Comparison of the Value of MSEv, WNSR and PSNR for the Test Images

It is clear from both Table1 and Fig. 4 that the proposed algorithm achieves consistently lower values of MSEv than the Lsmb algorithm, and with the increase in cluster partition, the value of MSEv is also decreases. We can be seen from Table1 and Fig. 4 that the proposed algorithm achieves consistently higher values of WSNR and PSNR than the Lsmb algorithm, and with the increase in cluster partition, the value of WSNR and PSNR are also increase.

\section{Conclusions}

This paper developed a based on the color image content digital halftoning. A detailed analysis on the proposed algorithm was presented. Analysis results show that the proposed algorithm produces better color halftone image quality when we increase the number of clustering, in particular details of the image. Simulation results also demonstrated that the proposed algorithm achieves consistently lower values of MSEv than the Lsmb algorithm. As the cluster partition increases, the value of MSEv decreases. And the proposed algorithm achieves consistently higher values of WSNR and PSNR than the color Lsmb algorithm. As the cluster partition increases, the value of WSNR and PSNR decreases.

\section{Acknowledgements}

This work was supported by Project 61302173 and 61461022 of the National Science Foundation of China and Foundation of Kunming University of Science and Technology under Grant 14118777, KKZ3201401003.

\section{References}

[1] C. A. Bouman: Digital Halftoning (Digital Image Processing, 2010).

[2] J.P. Allebach, Ed:Selected Papers on Digital Halftoning (SPIE:Bellingham,1999).

[3] Q. Lin and J. Allebach:Electronic Imaging Science and Technology, San Jose, CA, 1998, p. 353

[4] M. Wang and K. Parker: J. Imag. Sci. Technol., vol. 44, no. 4, p. 360

[5]D. Lau, A. Khan, and G. Arce: J. Opt. Soc. Amer. A, vol. 19, p. 2203

[6] T. Flohr and B. Kolpatzik: in Proc. SPIE Human Vision, Visual Processing, and Digital Display IV, vol. 1913, 1993, p. 270

[7] A.Ufuk Agar and Jan P. Allebach:IEEE Trans. Image Process Vol. 14(2005) ,p. 1945

[8] T. N. Pappas and D. L. Neuhoff: IEEE Trans. Image Processing, vol. 4, p. 66

[9] X. Zhang, D.A. Silverstein, J.E. Farrell, and B.A. Wandell: in IEEE COMP-CON97 Symp. Dig., 1997, p. 44. 